\title{
Stability of group homomorphisms in the compact-open topology
}

\author{
PAVOL Zlatoš ${ }^{1}$
}

\begin{abstract}
We introduce the notion of stability of continuous homomorphisms of topological groups with respect to the compact-open topology on the space of all continuous functions between them and prove that the characters of any locally compact abelian group are stable in this sense. Using this notion we also generalize a global stability result on continuous homomorphisms between compact groups to a local version for continuous homomorphisms from any locally compact group to an arbitrary topological group and show the necessity of the assumptions of the theorem through some counterexamples. Finally, we separately discuss the special cases of metrizable and discrete groups, respectively. As an application of the latter we prove a purely algebraic result on extendability of finite partial functions to group homomorphisms.
\end{abstract}

2000 Mathematics Subject Classification 22D12, 22B99 (primary); 22D10, 54H11, 54J05 (secondary)

Keywords: topological group, locally compact, compact-open topology, continuous homomorphism, character, stability

\section{Introduction}

Informally, a property of mappings between two structures of certain kind is stable if any mapping almost fulfilling the property is close to a mapping having that property. The problem of $\varepsilon$-stability of additive functions $\mathbb{R} \rightarrow \mathbb{R}$, as well as its generalization to mappings between arbitrary metrizable groups, was originally formulated by Ulam [19], [20]. Since then the topic has grown into an active research area—see Forti [5], Hyers-Isac-Rassias [10], Hyers-Rassias [11] and Rassias [18] for surveys of further development.

One can expect-even for the homomorphy property - that making precise the vague notions like "almost fulfilling the property" and "being close to" it is possible to

${ }^{1}$ Research supported by the VEGA grant 1/0588/09 
obtain various notions of stability. Nonetheless, it seems that so far the stability of continuous group homomorphisms between topological groups $G$ and $H$ was studied almost exclusively with respect to the topology of uniform convergence, though the last notion did not necessarily occur explicitly in such accounts. On the other hand, there are good reasons - at least for a locally compact group $G$ - to rate the compactopen topology (i e, the topology of uniform convergence on compact sets) as the most suitable topology on the set $\mathcal{C}(G, H)$ of all continuous function $G \rightarrow H$ (cf Engelking $[4, \S 3.4]$ ). Not speaking about locally compact abelian groups (briefly, LCA groups) for which it is the compact-open topology on the dual group of $G$ enabling to establish the celebrated Pontryagin-van Kampen duality (see Pontryagin [17], Morris [15]).

In this paper we systematically distinguish the global stability, ie, the stability with respect to the topology of uniform convergence, and the stability on compacts, i e, the stability with respect to the compact-open topology. As we shall see, within the latter framework it is natural to deal not just with everywhere defined mappings $G \rightarrow H$ but also with partial mappings $X \rightarrow H$ for various subsets $X \subseteq G$. Using two older known results as well as a more recent one (marked as Theorems A, B and C) we will establish a kind of stability result by proving that characters of any LCA group are stable on compacts. This is more in spirit of the Pontryagin-van Kampen duality theory (where the duals of LCA groups are endowed with the compact-open topology) than the global stability of characters of LCA groups proved in Cabello Sánchez [2] and Mačaj-Zlatoš [14].

For a compact group $G$ the two notions of stability coincide. However, as shown in Špakula, Zlatoš [21], even for compact abelian groups $G$ and $H$ continuous homomorphisms $G \rightarrow H$ need not be stable. In that paper a stability result for homomorphisms between compact groups was obtained introducing a kind of controlled continuity by means of a "continuity scale." Using the compact-open topology, we generalize this result to continuous homomorphisms from any locally compact group $G$ to an arbitrary topological group $H$. The theorem can be proved using the Arzèla-Ascoli theorem and projective limits of the system of groups of continuous functions $\mathcal{C}(X, H)$ with restriction maps over the directed set $\left(\mathcal{K}_{G}, \subseteq\right)$ of all compact subsets of $G$. However, we give a considerably shorter proof using nonstandard analysis. We also present two counterexamples showing that the assumptions of the theorem, needed in order either the Arzèla-Ascoli theorem or the nonstandard analysis methods to apply, are indeed necessary. At the same time, by a minor modification of the first counterexample we show that the global version of this stability theorem fails for locally compact $G$.

Finally, we separately discuss the special cases of metrizable and discrete groups, respectively. In particular, regarding every algebraic group as a locally compact group 
under the discrete topology, we prove a purely algebraic corollary about extendability of partial functions with finite domain to homomorphisms.

\section{The global stability and the stability on compacts}

In what follows the term topological group always means a group endowed with a Hausdorff topology in which both the operations of group multiplication and taking inverses are continuous. The topology of a topological group $G$ is completely described by the filter $\mathcal{O}_{G}=\mathcal{O}_{G}(1)$ of neighborhoods of the unit element $1 \in G$ : the filter $\mathcal{O}_{G}(x)$ of neighborhoods of a general element $x \in G$ is formed by the sets $x U$, where $U \in \mathcal{O}_{G}$. For all unexplained notions and basic results on topological groups the reader is referred to Pontryagin [17] or Morris [15].

The hooked arrow $f: G \hookrightarrow H$ denotes that $f$ is a partial mapping from $G$ to $H$, i e, $f: D \rightarrow H$ where $D=\operatorname{dom} f \subseteq G$.

Our basic notions are summed up in the following:

Definition 1 Let $G, H$ be arbitary groups, $X \subseteq G$ and $1 \in U \subseteq H$.

- Two partial mappings $f, g: G \hookrightarrow H$ are $U$-close on $X$ if for all $x \in X$

$$
X \subseteq \operatorname{dom} f \cap \operatorname{dom} g \quad \text { and } \quad f(x)^{-1} g(x) \in U .
$$

- A partial mapping $f: G \hookrightarrow H$ is called an $(X, U)$-homomorphism if $X \subseteq \operatorname{dom} f$ and for all $x, y \in X$ the condition $x y \in X$ implies

$$
f(x y)^{-1} f(x) f(y) \in U .
$$

- A partial mapping $f: G \hookrightarrow H$ is called a partial $U$-homomorphism if it is a $(\operatorname{dom} f, U)$-homomorphism.

- A (total) mapping $f: G \rightarrow H$ is called a $U$-homomorphism if it is a $(G, U)$-homomorphism.

If the topology of $H$ is induced by a (left, say) invariant metric $d$ and $\varepsilon>0$ then the notions like $\varepsilon$-close on $X,(X, \varepsilon)$-homomorphism, partial $\varepsilon$-homomorphism or $\varepsilon$-homomorphism mean $V_{\varepsilon}$-close on $X,\left(X, V_{\varepsilon}\right)$-homomorphism, partial $V_{\varepsilon}$-homomorphism or $V_{\varepsilon}$-homomorphism, respectively, where

$$
V_{\varepsilon}=\{v \in H ; d(v, 1) \leq \varepsilon\} \in \mathcal{O}_{H} .
$$

In the next definition we make precise and distinguish the two notions of stability, preliminarily discussed in the abstract and introduction. By $\mathcal{K}_{G}$ we denote the set of all compact subsets of $G$. 
Definition 2 Let $G, H$ be topological groups.

- Continuous homomorphisms $G \rightarrow H$ are globally stable if for every $V \in \mathcal{O}_{H}$ there is a $U \in \mathcal{O}_{H}$ such that every continuous $U$-homomorphism $f: G \rightarrow H$ is $V$-close on $G$ to some continuous homomorphism $\varphi: G \rightarrow H$.

- Continuous homomorphisms $G \rightarrow H$ are stable on compacts if for all $D \in \mathcal{K}_{G}$, $V \in \mathcal{O}_{H}$ there is a $C \in \mathcal{K}_{G}$ and a $U \in \mathcal{O}_{H}$ such that $D \subseteq C$ and every continuous $(C, U)$-homomorphism $f: G \hookrightarrow H$ is $V$-close on $D$ to some continuous homomorphism $\varphi: G \rightarrow H$.

While global stability deals with uniform approximability of continuous mappings $G \rightarrow H$ by continuous homomorphisms, stability on compacts deals with approximate extendability of continuous partial mappings $G \hookrightarrow H$ to continuous homomorphisms $G \rightarrow H$. In particular, for discrete $H$ the notion of global stability makes no sense while the stability on compacts still does: it deals with extendability of (continuous) partial mappings $G \hookrightarrow H$ to (continuous) homomorphisms $G \rightarrow H$.

Also notice that concerning logical strength the two notions are incomparable: proving global stability requires to derive stronger conclusions from stronger assumptions; on the other hand, in order to prove stability on compacts it is enough to derive weaker conclusions, however with weaker assumptions at ones disposal.

\section{Stability of characters of LCA groups}

Let us recall from Pontryagin [17] (cf also Morris [15]) that a continuous homomorphism of an LCA group $G$ into the multiplicative group of complex units $\mathbb{T}$ is called a character of $G$. The characters of $G$ with pointwise multiplication and the compactopen topology form an LCA group $\widehat{G}$, called the dual group of $G$, and the canonical mapping $G \rightarrow \widehat{\widehat{G}}$ is an isomorphisms of topological groups. We will also need the following structure theorem ([17], [15]), in a formulation emphasizing an additional point relevant for our purpose.

Theorem A Let $G$ be an LCA group generated by a compact symmetric neighborhood $V \in \mathcal{O}_{G}$. Then there exists a discrete finitely generated subgroup $A \subseteq G$ and an integer $m \geq 1$ such that $A \cap V=\{1\}, G / A$ is compact and $G=A V^{m}$.

Proof Indeed, the existence of a subgroup $A \subseteq G$ such that $A \cap V=\{1\}$ (already implying that $A$ is discrete), $G / A$ is compact and even $A \cong \mathbb{Z}^{k}$ for some $k \in \mathbb{N}$ 
is the point of proposition 33 in Morris [15]. Then the compact abelian group $G / A$ is generated by the compact symmetric neighborhood $V / A=A V / A \in \mathcal{O}_{G / A}$, i e, $G / A=\bigcup_{m=1}^{\infty}(V / A)^{m}$. Hence $G / A=(V / A)^{m}$ for some $m$, yielding $G=A V^{m}$.

Characters of LCA groups are globally stable-as LCA groups are amenable (see e g Pier [16]), this is a direct consequence of the following more general result proved in Cabello Sánchez [2] and (independently but later on) also in Mačaj-Zlatoš [14], slightly improving a special case of a theorem due to Kazhdan [12]. For convenience' sake we use the angular invariant metric

$$
\left|\arg \frac{a}{b}\right|=2 \arcsin \frac{|a-b|}{2}
$$

on $\mathbb{T}$ which is equivalent to the usual invariant euclidian metric $|a-b|$ inducing the topology of $\mathbb{T}$.

Theorem B Let $G$ be an amenable group. If $0<\varepsilon<\pi / 2$ then for every $\varepsilon$-homomorphism $f: G \rightarrow \mathbb{T}$ there exists a homomorphism $\varphi: G \rightarrow \mathbb{T}$ such that

$$
\left|\arg \frac{\varphi(x)}{f(x)}\right| \leq \varepsilon
$$

for each $x \in G$. Moreover, if $f$ is continuous then one can assume the same for $\varphi$.

Every algebraic abelian group $G$ can be viewed as an LCA group under the discrete topology, with compact subsets of $G$ being exactly the finite ones. Then the characters of discrete abelian groups are stable on finite sets. This is a direct consequence of the following more specific result proved in Mačaj, Zlatoš [14]. The number of elements of a finite set $S \subseteq G$ is denoted by \# $S$ and $\langle S\rangle_{n}$ denotes the subset of $G$ generated in at most $n$ steps from the elements of $S$ and $S^{-1}$, i e,

$$
\langle S\rangle_{n}=\left(S \cup S^{-1} \cup\{1\}\right)^{n} .
$$

Theorem $\mathbf{C}$ Let $0<\delta<\varepsilon \leq \pi / 2$ and $q \geq 1$ be an integer. Then there exists an integer $n \geq 1$ (depending just on $\delta, \varepsilon$ and $q$ ) such that for any (discrete) abelian group $G$, a set $S \subseteq G$, satisfying $\# S \leq q$, and a partial $\delta$-homomorphism $f:\langle S\rangle_{n} \rightarrow \mathbb{T}$ there is a character $\varphi: G \rightarrow \mathbb{T}$ such that for each $s \in S$

$$
\left|\arg \frac{\varphi(s)}{f(s)}\right|<\varepsilon
$$


The proof in Mačaj-Zlatoš [14] is based on Theorem B and a nonstandard-analysis result by Gordon [7] (cf also Gordon [8]) implying that for each character $g: X \rightarrow \mathbb{T}$ of a countable subgroup $X$ of an ultraproduct $G=\prod_{i \in \mathbb{N}} G_{i} / \mathcal{D}$ of a system of abelian groups $G_{i}$ there exists an internal character $\gamma: G \rightarrow \mathbb{T}^{\mathbb{N}} / \mathcal{D}$ such that $\gamma(x) \approx g(x)$ for each $x \in X .{ }^{2}$ However, these proofs do not give an estimate in terms of $\delta, \varepsilon$ and $q$ how big the $n$ in Theorem $\mathrm{C}$ has to be.

The main goal of this section is to prove that characters of locally compact groups are stable on compacts. First we establish a rather detailed technical version of this fact for compactly generated LCA groups. The proof heavily depends on Theorem $\mathrm{C}$ by means of which the situation can be reduced to Theorem B.

Theorem 1 Let $0<3 \delta<\varepsilon \leq \pi / 2$ and $q \geq 1$ be an integer. Then there exists an integer $n \geq 1$ (depending just on $\delta, \varepsilon$ and $q$ ) with the following property:

If $G$ is a locally compact abelian group, $A$ is a discrete subgroup of $G$ generated by a finite set $S$ and $V \in \mathcal{O}_{G}$ is a compact symmetric neighborhood, satisfying $G=A V, A \cap V^{3} \subseteq S$ and $\# S \leq q$, then for every continuous partial $\delta$-homomorphism $f:\langle S\rangle_{n} V^{2} \rightarrow \mathbb{T}$ there is a character $\varphi: G \rightarrow \mathbb{T}$ such that

$$
\begin{aligned}
& \left|\arg \frac{\varphi(x)}{f(x)}\right|<\varepsilon, \quad \text { for } x \in V, \\
& \left|\arg \frac{\varphi(s)}{f(s)}\right|<\varepsilon+2 \delta, \quad \text { for } s \in S .
\end{aligned}
$$

Proof As $3 \delta<\varepsilon$, there is an $\eta>\delta$ such that $3 \eta<\varepsilon$ still holds. Let $n$ be the integer guaranteed to $\delta, \eta$ and $q$ by Theorem C. Assume that $G, A, S$ and $V$ satisfy the above assumptions, and $f:\langle S\rangle_{n} V^{2} \rightarrow \mathbb{T}$ is a continuous partial $\delta$ homomorphism. According to the choice of $n$, there is a homomorphism $\gamma: A \rightarrow \mathbb{T}$ such that $|\arg (\gamma(s) / f(s))| \leq \eta$ for each $s \in S$.

As $G=A V$, there are functions $x \mapsto x_{A}: G \rightarrow A$ and $x \mapsto x_{V}: G \rightarrow V$ such that $x=x_{A} x_{V}$ for each $x \in G$ and $x_{V}=x$ whenever $x \in V$. Let us define the function $h: G \rightarrow \mathbb{T}$ by

$$
h(x)=\gamma\left(x_{A}\right) f\left(x_{V}\right)
$$

\footnotetext{
${ }^{2}$ This is to say that $\gamma=\left(\gamma_{i}\right) / \mathcal{D}$ where each $\gamma_{i}: G_{i} \rightarrow \mathbb{T}$ is a character of $G_{i}$ and for all $x=\left(x_{i}\right) / \mathcal{D} \in X$ and $\varepsilon>0$ we have $\left\{i \in \mathbb{N} ;\left|\arg \left(\gamma_{i}\left(x_{i}\right) / g(x)\right)\right|<\varepsilon\right\} \in \mathcal{D}$.
} 
for $x \in G$. Obviously, $h(x)=f(x)$ for $x \in V$. For $s \in S$ we have $s_{V}=s s_{A}^{-1} \in$ $A \cap V \subseteq S$, and

$$
\frac{h(s)}{f(s)}=\frac{\gamma\left(s_{A}\right) f\left(s_{V}\right)}{f(s)}=\frac{\gamma(s)}{f(s)} \cdot \frac{f\left(s_{V}\right)}{\gamma\left(s_{V}\right)},
$$

hence $|\arg (h(s) / f(s))| \leq 2 \eta$.

Let us estimate the distance between $h(x y)$ and $h(x) h(y)$ for $x, y \in G$. First, observe that from the two-fold expression

$$
x y=(x y)_{A}(x y)_{V}=x_{A} x_{V} y_{A} y_{V}
$$

it follows that for the element

$$
z=(x y)_{A} x_{A}^{-1} y_{A}^{-1}=x_{V} y_{V}(x y)_{V}^{-1}
$$

we have $z, z^{-1} \in A \cap V^{3} \subseteq S$. Then both $f(z)$ and $f\left(x_{V} y_{V}\right)$ are defined. As $\gamma$ is a homomorphism, by a straightforward expansion we get

$$
\begin{aligned}
\frac{h(x) h(y)}{h(x y)}= & \frac{\gamma\left(x_{A}\right) f\left(x_{V}\right) \gamma\left(y_{A}\right) f\left(y_{V}\right)}{\gamma(x y)_{A} f(x y)_{V}} \\
= & \frac{\gamma\left(x_{A}\right) \gamma\left(y_{A}\right)}{\gamma(x y)_{A}} \cdot \frac{f\left(x_{V}\right) f\left(y_{V}\right)}{f\left(x_{V} y_{V}\right)} \cdot \frac{f\left(x_{V} y_{V}\right)}{f(x y)_{V} f\left(x_{V} y_{V}(x y)_{V}^{-1}\right)} \\
& \quad \cdot \frac{f\left(x_{V} y_{V}(x y)_{V}^{-1}\right)}{\gamma\left(x_{V} y_{V}(x y)_{V}^{-1}\right)} \cdot \gamma\left(x_{V} y_{V}(x y)_{V}^{-1}\right) \\
= & \gamma\left(z^{-1}\right) \cdot \frac{f\left(x_{V}\right) f\left(y_{V}\right)}{f\left(x_{V} y_{V}\right)} \cdot \frac{f\left(x_{V} y_{V}\right)}{f(x y)_{V} f(z)} \cdot \frac{f(z)}{\gamma(z)} \cdot \gamma(z) \\
= & \frac{f\left(x_{V}\right) f\left(y_{V}\right)}{f\left(x_{V} y_{V}\right)} \cdot \frac{f\left(x_{V} y_{V}\right)}{f\left(x_{V} y_{V} z^{-1}\right) f(z)} \cdot \frac{f(z)}{\gamma(z)} .
\end{aligned}
$$

Since $f$ is a partial $\delta$-homomorphism and $f$ and $\gamma$ are $\eta$-close on $S$, the inequality

$$
\left|\arg \frac{h(x) h(y)}{h(x y)}\right| \leq 2 \delta+\eta
$$

immediately follows, showing that $h$ is a $(2 \delta+\eta)$-homomorphism. Since $G$ is amenable, according to Theorem B, there is a homomorphisms $\varphi: G \rightarrow \mathbb{T}$ such that $|\arg (\varphi(x) / h(x))| \leq 2 \delta+\eta$ for each $x \in G$. As $h(x)=f(x)$ for $x \in V$,

$$
\left|\arg \frac{\varphi(x)}{f(x)}\right| \leq 2 \delta+\eta<\varepsilon
$$

For $s \in S$ we have

$$
\frac{\varphi(s)}{f(s)}=\frac{\varphi(s)}{h(s)} \cdot \frac{h(s)}{f(s)}
$$


yielding the estimate

$$
\left|\arg \frac{\varphi(s)}{f(s)}\right| \leq 2 \delta+3 \eta<\varepsilon+2 \delta
$$

It remains to prove that $\varphi$ is continuous. Since $\varphi$ is a homomorphism, to this end it is enough to show that there is some neighborhood $U \in \mathcal{O}_{G}$ such that $|\arg \varphi(x)|<2 \pi / 3$ for all $x \in U .{ }^{3}$ From $3 \delta<\varepsilon \leq \pi / 2$ it follows $\varepsilon+\delta<\pi / 2+\pi / 6=2 \pi / 3$. Let us pick some $\sigma>0$ such that $\varepsilon+\delta+\sigma<2 \pi / 3$ still holds. As $f$ is continuous on $V$ (in particular in $1 \in V)$, there is a $U \in \mathcal{O}_{G}$ such that $U \subseteq V$ and $|\arg (f(x) / f(1))| \leq \sigma$ for each $x \in U$. Then

$$
\varphi(x)=\frac{\varphi(x)}{f(x)} \cdot \frac{f(x)}{f(1)} \cdot f(1),
$$

and, as $|\arg f(1)| \leq \delta$, we finally get

$$
|\arg \varphi(x)|<\varepsilon+\sigma+\delta<\frac{2 \pi}{3} .
$$

Theorem 2 Let $G$ be a locally compact abelian group. Then the characters of $G$ are stable on compacts.

Proof Let $K \subseteq G$ be a compact set and $0<\varepsilon \leq \pi / 2$. We will show that there is a compact neighborhood $U \in \mathcal{O}_{G}$ and a $\delta>0$ such that $K \subseteq U, \delta<\varepsilon$ and for every continuous partial $\delta$-homomorphism $f: U \rightarrow \mathbb{T}$ there is a continuous character $\varphi: G \rightarrow \mathbb{T}$ such that for all $x \in K$

$$
\left|\arg \frac{\varphi(x)}{f(x)}\right|<\varepsilon .
$$

$K$, being compact, is contained in some compact symmetric neighborhood of 1 , so that we can assume, without loss of generality, that $K$ itself is such neighborhood. Let $H$ be the subgroup of $G$ generated by $K$. Then $H$ is both open and closed and, according to Theorem A, there is a discrete closed subgroup $A \subseteq H$ generated by some finite set $S_{0} \subseteq A$, such that $A \cap K=\{1\}$, the quotient $H / A$ is compact and $H=A K^{m}$ for some integer $m \geq 1$. Then $V=K^{m}$ is a compact symmetric neighborhood of 1 , again. As $A$ is discrete, the set $A \cap V^{3}$ is finite, hence the set $S=S_{0} \cup\left(A \cap V^{3}\right)$ is also finite and generates $A$. Denote $q=\# S$. Now, choose any $\delta<\varepsilon / 3$ and fix the $n$ guaranteed

\footnotetext{
${ }^{3}$ Indeed, as $\{1\}$ is the only subgroup of $\mathbb{T}$ contained in the arc $\{c \in \mathbb{T} ;|\arg c|<2 \pi / 3\}$, whenever $W \in \mathcal{O}_{G}$ satisfies $W^{k} \subseteq U$ we already have $|\arg \varphi(x)|<2 \pi / 3 k$ for each $x \in W$.
} 
to $\delta$, $\varepsilon$ and $q$ in Theorem 1. Finally, put $U=\langle S\rangle_{n} V^{2}$. As $\langle S\rangle_{n}$ is finite and $V^{2}$ is compact, $U$ is compact, as well.

Assume that $f: U \rightarrow \mathbb{T}$ is a continuous partial $\delta$-homomorphism. Then, due to the choice of $n$ and Theorem 1, there is a continuous character $\psi: H \rightarrow \mathbb{T}$ such that

$$
\left|\arg \frac{\psi(x)}{f(x)}\right|<\varepsilon
$$

for all $x \in V \supseteq K$. By the divisibility property of $\mathbb{T}, \psi$ can be extended to a continuous character $\varphi: G \rightarrow \mathbb{T}$ (cf Pontryagin [17, Theorem 55]).

$\mathbb{T}=\mathrm{U}(1)$ is just the first one from among the unitary groups $\mathrm{U}(n)$. Thus Theorem 2 naturally raises the question of stability on compacts of continuous homomorphisms $G \rightarrow \mathrm{U}(n)$ for $n \geq 1$ for various classes of (in general nonabelian) locally compact groups $G$ (discrete, amenable, unimodular, arbitrary, ....). In view of Kazhdan's theorem [12, Theorem 1], establishing global stability of representations $G \rightarrow \mathrm{U}(X)$ of amenable groups $G$ in the group $\mathrm{U}(X)$ of unitary operators on an arbitrary Hilbert space $X$, it could be interesting to examine the issue of stability on compacts in this situation, as well.

\section{Stability of homomorphisms from locally compact groups}

There are infinitely many pairs of compact metrizable abelian groups $G$ and $H$ such that the continuous homomorphisms $G \rightarrow H$ are not globally stable. The following example is taken from Špakula-Zlatoš [21, Theorem 1].

Example 1 Let $p$ be any prime number, $G=\prod_{k=1}^{\infty} \mathbb{Z} /\left(p^{k}\right)$ be the direct product of the finite cyclic groups $\mathbb{Z} /\left(p^{k}\right)$ and $H=\mathbb{Z}_{p}$ be the group of $p$-adic integers with its usual norm $|\cdot|_{p}$. Then the function $f_{n}: G \rightarrow H$ sending a sequence $\left(x_{k}\right) \in G$ to its $n$th term $x_{n} \in\left\{0,1, \ldots, p^{n}-1\right\} \subseteq \mathbb{Z}_{p}$ is a continuous $p^{-n}$-homomorphism. However, as $\mathbb{Z}_{p}$ has no torsion, the only homomorphism $G \rightarrow H$ factoring through the projection $G \rightarrow \mathbb{Z} /\left(p^{n}\right)$ is the trivial one. As a consequence,

$$
\sup _{x \in G}\left|f_{n}(x)-\varphi(x)\right|_{p} \geq 1
$$

for each $n$ and every homomorphism $\varphi: G \rightarrow H$, and not just a continuous one (see Špakula-Zlatoš [21] for details).

Positive results can be obtained introducing some control of continuity of partial mappings $G \hookrightarrow H$. 
Definition 3 Let $G$ and $H$ be topological groups and $\mathcal{B}$ be a basis of the filter of neighborhoods $\mathcal{O}_{H}$.

- A $(G, H)$-continuity scale is any mapping $\Gamma: G \times \mathcal{B} \rightarrow \mathcal{P}(G)$ assigning to each pair $(x, U) \in G \times \mathcal{B}$ a neighborhood $\Gamma(x, U)$ of the point $x$ in $G$.

- A partial mapping $f: G \hookrightarrow H$ is $\Gamma$-continuous with respect to a $(G, H)$-continuity scale $\Gamma: G \times \mathcal{B} \rightarrow \mathcal{P}(G)$ if for all $x, y \in \operatorname{dom} f$ and $U \in \mathcal{B}$ the condition $y \in \Gamma(x, U)$ implies $f(y) \in f(x) U$.

The main reason for introducing the above notions is the following fact: A family of partial functions $G \hookrightarrow H$ is equicontinuous if and only if it is $\Gamma$-continuous with respect to some $(G, H)$-continuity scale $\Gamma .{ }^{4}$

For compact groups, where continuity already implies uniform continuity, it is enough to define a continuity scale as a mapping $\Gamma: \mathcal{B} \rightarrow \mathcal{O}_{H}$; then $f: G \hookrightarrow H$ is (uniformly) $\Gamma$-continuous if and only $x^{-1} y \in \Gamma(U)$ implies $f(x)^{-1} f(y) \in U$, for all $x, y \in \operatorname{dom} f$ and $U \in \mathcal{B}$.

The following global stability result was proved in Špakula, Zlatoš [21, Theorem 2].

Theorem D Let $G, H$ be compact topological groups and $\Gamma$ be a $(G, H)$-continuity scale. Then for each $V \in \mathcal{O}_{H}$ there is a $U \in \mathcal{O}_{H}$ such that every $\Gamma$-continuous $U$-homomorphism $f: G \rightarrow H$ is $V$-close on $G$ to a continuous homomorphism $\varphi: G \rightarrow H$.

Under some necessary minor modifications Theorem D can be generalized to a stability result for continuous homomorphisms between compact topological universal algebras (Zlatoš [23]).

Example 1 shows that one cannot dispense with the continuity scale $\Gamma$. Moreover, regarding $G=\prod_{k=1}^{\infty} \mathbb{Z} /\left(p^{k}\right)$ as a discrete (hence locally compact) topological group and taking any basis $\mathcal{B}$ of the filter of neighborhoods of $0 \in H=\mathbb{Z}_{p}$, the assignment $(x, U) \mapsto\{x\}$ for $x \in G, U \in \mathcal{B}$ defines the discrete continuity scale $\Delta: G \times \mathcal{B} \rightarrow \mathcal{P}(G)$. Obviously, every function $f: G \rightarrow H$ is $\Delta$-continuous. Then Example 1, modified in this way, shows at the same time that Theorem D does not hold for locally compact groups $G$-not even in the case of $G$ discrete abelian and $H$ compact abelian or discrete abelian.

\footnotetext{
${ }^{4}$ The "if part" is trivial while the "only if part" is an obvious consequence of the definition of equicontinuity (see, e g, Engelking [4, pp. 542]) and the axiom of choice.
} 
In order to generalize Theorem D to locally compact groups $G$ and arbitrary topological groups $H$ we have to replace the topology of uniform convergence on $\mathcal{C}(G, H)$ by the topology of uniform convergence on compacts. However, the assumption of compactness of the group $H$ requires a substitute of a different kind.

Definition 4 Let $X, Y$ be arbitrary sets.

- A bounding relation from $X$ to $Y$ is any binary relation $R \subseteq X \times Y$ such that the set $R[x]=\{y \in Y ;(x, y) \in R\}$ is nonempty for each $x \in X$.

- A partial mapping $f: X \rightarrow Y$ is $R$-bounded with respect to the bounding relation $R$ if $f \subseteq R$, i e, if $f(x) \in R[x]$ for each $x \in \operatorname{dom} f$.

- A bounding relation $R \subseteq X \times Y$ is stalkwise finite if all the stalks $R[x]$ are finite.

- If $Y$ is additionally a topological space then a bounding relation $R \subseteq X \times Y$ is called stalkwise compact if all the stalks $R[x]$ are compact subsets of $Y$.

Now we are ready to formulate and prove the announced generalization of Theorem D. Our proof makes use of some methods of nonstandard analysis; the reader can consult e g Davis [3], or Arkeryd-Cutland-Henson [1], mainly the parts by Henson [9] and Loeb [13].

Theorem 3 Let $G$ be a locally compact and $H$ be an arbitrary topological group. Let $\Gamma$ be a $(G, H)$-continuity scale and $R \subseteq G \times H$ be a stalkwise compact bounding relation. Then for each pair $D \in \mathcal{K}_{G}, V \in \mathcal{O}_{H}$ there exists a pair $C \in \mathcal{K}_{G}, U \in \mathcal{O}_{H}$ such that $D \subseteq C$ and every $\Gamma$-continuous $R$-bounded $(C, U)$-homomorphism $f: G \hookrightarrow H$ is already $(D, V)$-close to some continuous homomorphism $\varphi: G \rightarrow H$.

Proof Assume the contrary and fix some topological groups $G, H$, with a $(G, H)$-continuity scale $\Gamma: G \times \mathcal{B} \rightarrow \mathcal{P}(G)$, where $\mathcal{B}$ is a basis of the neighborhood filter $\mathcal{O}_{H}$, a stalkwise compact bounding relation $R \subseteq G \times H$ and a pair $D \in \mathcal{K}_{G}, V \in \mathcal{O}_{H}$, witnessing it.

Then for any pair $C \in \mathcal{K}_{G}, U \in \mathcal{B}$, such that $D \subseteq C$, there is a $\Gamma$-continuous $R$-bounded $(C, U)$-homomorphism $f: C \rightarrow H$ such that for every continuous homomorphism $\varphi: G \rightarrow H$ one can find an $x \in D$ satisfying $f(x)^{-1} \varphi(x) \notin V$.

Let us embed the situation into a sufficiently saturated nonstandard universe, ${ }^{5}$ via the canonic elementary embedding *. As usual, $G$ and $H$ are identified with subgroups

\footnotetext{
${ }^{5}$ In precise terms this is to say that the universe is $\kappa$-saturated for a cardinal number $\kappa$ bigger than $\max \left(\operatorname{card} \mathcal{K}_{0}\right.$, card $\left.\mathcal{B}_{0}, \aleph_{0}\right)$, where $\mathcal{K}_{0}, \mathcal{B}_{0}$ are some cofinal subsets of minimal cardinality in $\left(\mathcal{K}_{G}, \subseteq\right),\left(\mathcal{O}_{H}, \supseteq\right)$, respectively.
} 
of their extensions ${ }^{*} G,{ }^{*} H$, respectively. The relation of infinitesimal nearness, both on ${ }^{*} G$ and ${ }^{*} H$, is denoted by $\approx$. An element $\xi \in{ }^{*} G$ is called nearstandard if $\xi \approx x$ for some $x \in G$. The subgroup of nearstandard elements in ${ }^{*} G$ is denoted by ns $\left({ }^{*} G\right)$. Obviously $G \subseteq \mathrm{ns}\left({ }^{*} G\right) \subseteq{ }^{*} G$. For $\xi \in \mathrm{ns}\left({ }^{*} G\right)$ we denote by ${ }^{\circ} \xi$ the standard part of $\xi$, i e, the unique element of $G$ infinitesimally close to $\xi$. Analogous terminology and notation applies to ${ }^{*} H$.

By the virtue of $\kappa$-saturation, there is a pair $E \in{ }^{*} \mathcal{K}_{G}, W \in{ }^{*} \mathcal{O}_{H}$ such ${ }^{*} C \subseteq E$ and $W \subseteq{ }^{*} U$ for all $C \in \mathcal{K}_{G}, U \in \mathcal{O}_{H}$, and an internal ${ }^{*} \Gamma$-continuous, ${ }^{*} R$-bounded $(E, W)$-homomorphism $F: E \rightarrow{ }^{*} H$. By transfer, for every internal ${ }^{*}$-continuous homomorphism $\Phi:{ }^{*} G \rightarrow{ }^{*} H$ there is a $\xi \in{ }^{*} D$ subject to $F(\xi)^{-1} \Phi(\xi) \notin{ }^{*} V$. As $G$ is locally compact, $\mathrm{ns}\left({ }^{*} G\right) \subseteq E$. By the compactness of $D$, each element of ${ }^{*} D$ is nearstandard, hence ${ }^{*} D \subseteq \mathrm{ns}\left({ }^{*} G\right) \subseteq E$.

As a consequence we have $F(\xi \eta) \approx F(\xi) F(\eta)$ for all $\xi, \eta \in \mathrm{ns}\left({ }^{*} G\right)$. Since all the stalks $R[x]$ are compact, $F(x) \in{ }^{*} R[x] \subseteq \mathrm{ns}\left({ }^{*} H\right)$ for $x \in G$. Thus the mapping $\varphi: G \rightarrow H$, where $\varphi(x)={ }^{\circ} F(x)$ for $x \in G$ is a well defined group homomorphism. From the ${ }^{*} \Gamma$-continuity of $F$ it easily follows that it is S-continuous, ${ }^{6}$ hence $F(\xi) \in \mathrm{ns}\left({ }^{*} H\right)$ for $\xi \in \mathrm{ns}\left({ }^{*} G\right)$. Thus we have the following commutative diagram:

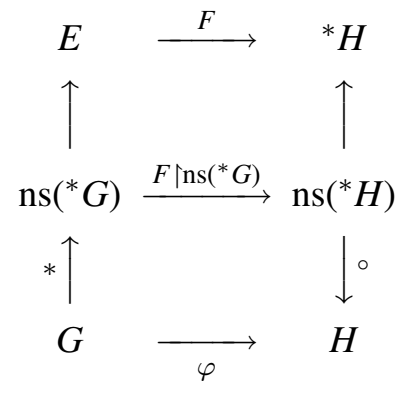

where the upper vertical arrows $\uparrow$ denote inclusion maps. As $F$ is $S$-continuous, $\varphi$ is continuous. Then the mapping ${ }^{*} \varphi:{ }^{*} G \rightarrow{ }^{*} H$ is an internal S-continuous and ${ }^{*}$-continuous group homomorphism, extending $\varphi$. For each $\xi \in \mathrm{ns}\left({ }^{*} G\right)$ we have $\xi \approx{ }^{\circ} \xi$, therefore

$$
F(\xi) \approx F\left({ }^{\circ} \xi\right) \approx{ }^{\circ} F\left({ }^{\circ} \xi\right)=\varphi\left({ }^{\circ} \xi\right)={ }^{*} \varphi\left({ }^{\circ} \xi\right) \approx{ }^{*} \varphi(\xi),
$$

contradicting $F(\xi)^{-1 *} \varphi(\xi) \notin^{*} V$ for some $\xi \in{ }^{*} D \subseteq \mathrm{ns}\left({ }^{*} G\right)$.

Similarly as Theorem D, also the last Theorem 3 admits a generalization to a stability result for continuous homomorphisms $A \rightarrow B$ between topological universal algebras,

${ }^{6}$ I.e., $\xi \approx \eta$ implies $F(\xi) \approx F(\eta)$ for all $\xi, \eta \in E$. 
with $A$ locally compact and $B$ completely regular. This result is proved in Zlatoš [22] using the Arzèla-Ascoli theorem and the projective limit of the system of algebras $\mathcal{C}(D, B) \subseteq B^{D}$ of continuous functions $D \rightarrow B$ defined on compact sets $D \subseteq A$ with the restriction maps $\mathcal{C}(E, B) \rightarrow \mathcal{C}(D, B)$ for $D \subseteq E$. The proof avoids nonstandard analysis completely but is considerably longer.

The next counterexample was suggested by M. Mačaj. As it follows from it, one cannot avoid the compact bounding relation $R$ in the formulation of Theorem 3-not even in the discrete abelian case.

Example 2 Consider the additive abelian groups $\mathbb{Q}$ of all rational numbers and $\mathbb{Z}$ of all integers as topological groups with the discrete topology. Then the singleton $\left\{\operatorname{Id}_{\mathbb{Z}}\right\}$, where $\mathrm{Id}_{\mathbb{Z}}$ is the identity relation on $\mathbb{Z}$, forms a basis of the discrete uniformity on $\mathbb{Z}$ and the assignment $\Delta\left(x, \operatorname{Id}_{\mathbb{Z}}\right)=\{x\}$, for $x \in \mathbb{Q}$, defines the discrete $(\mathbb{Q}, \mathbb{Z})$-continuity scale. Obviously, every partial function $\mathbb{Q} \hookrightarrow \mathbb{Z}$ is $\Delta$-continuous.

The compact subsets of $\mathbb{Q}$ are exactly the finite ones and each finite set $F \subseteq \mathbb{Q}$ generates a cyclic subgroup $\langle F\rangle$ of $\mathbb{Q}$ isomorphic to the free abelian group $\mathbb{Z}$. Thus for any finite $F \subseteq \mathbb{Q}$ containing an element $a \neq 0$ there is even a homomorphism $h:\langle F\rangle \rightarrow \mathbb{Z}$ such that $h(a) \neq 0$ (and not only a nonzero partial homomorphism $F \rightarrow \mathbb{Z}$ ). However, as well known, there is no homomorphism $\mathbb{Q} \rightarrow \mathbb{Z}$ except for the trivial one.

For metrizable topological groups $G, H$ endowed with (left) invariant metrics $\rho$, $\sigma$, respectively, a $(G, H)$-continuity scale can be more transparently defined as a sequence of pairs $\Gamma=\left(\left(\gamma_{k}, \eta_{k}\right)\right)_{k \in \mathbb{N}}$ where $\left(\eta_{k}\right)_{k}$ is a decreasing sequence of positive reals converging to 0 and each $\gamma_{k}$ is a function $G \rightarrow \mathbb{R}^{+}$. A mapping $f: G \hookrightarrow H$ is $\Gamma$-continuous if for all $k \in \mathbb{N}, x, y \in \operatorname{dom} f$ the condition $\rho(x, y) \leq \gamma_{k}(x)$ implies $\sigma(f(x), f(y)) \leq \eta_{k}$. Then the metric version of Theorem 3 reads as follows:

Corollary 1 Let $G$ and $H$ be topological groups with (left) invariant metrics $\rho$ and $\sigma$, respectively, and $G$ be locally compact. Let $\Gamma=\left(\left(\gamma_{k}, \eta_{k}\right)\right)_{k}$ be a $(G, H)$-continuity scale and $R \subseteq G \times H$ be a stalkwise compact bounding relation. Then for each pair $D \in \mathcal{K}_{G}, \varepsilon>0$ there exists a pair $C \in \mathcal{K}_{G}, \delta>0$ such that $D \subseteq C$ and every $\Gamma$-continuous $R$-bounded $(C, \delta)$-homomorphism $f: G \hookrightarrow H$ is already $(D, \varepsilon)$-close to some continuous homomorphism $\varphi: G \rightarrow H$.

Regarding any algebraic group as a locally compact topological group with the discrete topology, Theorem 3 has some corollaries on extending partial homomorphisms to homomorphisms. 
Corollary 2 Let $G, H$ be arbitrary groups and $R \subseteq G \times H$ be a stalkwise finite bounding relation. Then to each finite set $D \subseteq G$ there exists a finite set $C \subseteq G$ such that $D \subseteq C$ and for every $R$-bounded partial homomorphism $f: C \rightarrow H$ there is a homomorphism $\varphi: G \rightarrow H$ such that $f(x)=\varphi(x)$ for all $x \in D$.

Under some additional assumptions on the groups $G$ and $H$ one can make a closer estimate of $C$ as the set generated from $D$ in certain finite number of steps.

A group $H$ is called injective in a class $\mathbf{C}$ of groups (in the category theoretical sense) if $H \in \mathbf{C}$ and for every group $G \in \mathbf{C}$ and its subgroup $G_{0} \in \mathbf{C}$ any homomorphism $\varphi_{0}: G_{0} \rightarrow H$ can be extended to a homomorphism $\varphi: G \rightarrow H$. The injectivity property is particularly important for abelian groups; it is well known that an abelian group is injective (with respect to the class of all abelian groups) if and only if it is divisible—see, e g Fuchs [6].

Corollary 3 Let $G, H$ be groups, $R \subseteq G \times H$ be a stalkwise finite bounding relation, and $D \subseteq G$ be a finite set. Assume that either $D$ generates $G$ or $H$ is injective with respect to some class of groups containing $G$. Then there is an $n \in \mathbb{N}$ such that every $R$-bounded partial homomorphism $f:\langle D\rangle_{n} \rightarrow H$ can be extended to a homomorphism $\varphi: G \rightarrow H$.

Proof a) Assume that $G$ is generated by $D$. Let $C \subseteq G$ be the set guaranteed to $D$ by corollary 2. As $C$ is finite and $G=\bigcup_{n}\langle D\rangle_{n}, C \subseteq\langle D\rangle_{n}$ for some $n$.

b) Assume that $H$ is injective with respect to $G$. Denote by $G_{0}=\langle D\rangle$ the subgroup of $G$ generated by $D$. Let $n \in \mathbb{N}$ be the number guaranteed to the finite set $D \subseteq G_{0}$ in part a) of the proof applied to the groups $G_{0}, H$. Then every $R$-bounded partial homomorphism $f:\langle D\rangle_{n} \rightarrow H$ extends to a homomorphism $\varphi_{0}: G_{0} \rightarrow H$. By the injectivity of $H, \varphi_{0}$ extends to a homomorphism $\varphi: G \rightarrow H$.

\section{References}

[1] L O Arkeryd, N J Cutland, C W Henson, (eds), Nonstandard Analysis, Theory and Applications, Kluwer Academic Publishers, Dordrecht-Boston-London (1997).

[2] F Cabello Sánchez, Pseudo-characters and almost multiplicative functionals, J. Math. Anal. Appl. 248 (2000) 275-289; doi:10.1006/jmaa.2000.6898.

[3] M Davis, Applied Nonstandard Analysis, John Wiley Sons, New York-London (1977).

[4] R Engelking, General Topology, Polish Scientific Publishers PWN, Warszawa (1977). 
[5] G L Forti, Hyers-Ulam stability of functional equations in several variables, Aequationes Math. 50 (1995) 143-190; doi:10.1007/BF01831117.

[6] L Fuchs, Infinite Abelian Groups, Academic Press, London-New York (1970).

[7] E I Gordon, Nonstandard analysis and locally compact abelian groups, Acta Appl. Math. 25 (1991) 221-239.

[8] E I Gordon, Nonstandard Methods in Commutative Harmonic Analysis, Translations of mathematical monographs vol. 164, Amer. Math. Soc., Providence, R. I. (1997).

[9] C W Henson, Foundations of nonstandard analysis: A gentle introduction to nonstandard extensions, from: [1] (1997) 1-49.

[10] D H Hyers, G Isac, T M Rassias, Stability of Functional Equations in Several Variables, Birkhäuser Verlag, Basel-Boston (1998).

[11] D H Hyers, T M Rassias, Approximate homomorphisms, Aequationes Math. 44 (1992) 125-153; doi:10.1007/BF01830975.

[12] D Kazhdan, On ع-representations, Israel J. Math. 43 (1982) 315-323.

[13] P A Loeb, Nonstandard analysis and topology, from: [1] (1997), 77-89.

[14] M Mačaj, P Zlatoš, Approximate extension of partial $\varepsilon$-characters of abelian groups to characters with application to integral point lattices, Indag. Math. 16 (2005) 237-250; doi:10.1016/S0019-3577(05)80026-6.

[15] S A Morris, Pontryagin Duality and the Structure of Locally Compact Abelian Groups, Cambridge University Press, London-New York-Melbourne (1977).

[16] J-P Pier, Amenable Locally Compact Groups, John Wiley \& Sons, New YorkChichester (1984).

[17] LS Pontryagin, Nepreryvnye grupy (Continuous Groups), Nauka, Moskva (1984).

[18] T M Rassias, On the stability of functional equations and a problem of Ulam, Acta Applicanda Math. 62 (2000) 23-130; doi:10.1023/A:1006499223572.

[19] S M Ulam, A Collection of Mathematical Problems, Interscience Publishers, New York (1960).

[20] S M Ulam, An anecdotal history of the Scottish Book, from: "R. D Mauldin (ed.), The Scottish Book", Birkhäuser Verlag, Basel-Boston (1981).

[21] J Špakula, P Zlatoš, Almost homomorphisms of compact groups, Illinois J. Math. 48 (2004) 1183-1189.

[22] P Zlatoš, Stability of homomorphisms in the compact-open topology, to appear in Algebra Universalis.

[23] P Zlatoš, Stability of homomorphisms between compact algebras, Acta Univ. Mathaei Belii, ser. Math. 15 (2009) 73-79.

Faculty of Mathematics, Physics and Informatics, Comenius University, Bratislava

zlatos@fmph. uniba.sk

Received: 6 October $2009 \quad$ Revised: 26 March 2010 